\title{
DESIGN DAYS BOOT CAMP 2.0: IMPROVEMENTS AND CONNECTIONS TO CEAB GRADUATE ATTRIBUTES
}

\author{
Jennifer Howcroft, Igor Ivkovic, Matthew J. Borland, and Maud Gorbet \\ Systems Design Engineering, University of Waterloo \\ jenny.howcroft@uwaterloo.ca, igor.ivkovic@uwaterloo.ca, mjborlan@uwaterloo.ca, mgorbet@uwaterloo.ca
}

\begin{abstract}
Engineering design is a critical skill that all engineering students are expected to learn and is often the focus of final year capstone projects and first-year cornerstone projects. In the Systems Design Engineering Department at the University of Waterloo, engineering design is introduced to the students during an intense twoday Design Days Boot Camp. Design Days was originally conceived of and run in Fall 2016. The Fall 2018 version, Design Days 2.0, included substantial improvements focused on adding two additional design activities and a writing activity, strengthening the connection with first year content, and providing a greater variety of team experiences. The methods of achieving the nine intended learning outcomes of Design Days 2.0 are discussed and connected to CEAB graduate attributes. This demonstrates that meaningful learning can be achieved during a two-day boot camp that will starts students on the path towards professional engineering. Other departments are encouraged to use the presented intended learning outcomes, graduate attributes connections, and Design Days 2.0 descriptions as a template for their own design boot camp. Finally, Design Days 2.0 inspired ideas for further improvements including the incorporation of a software-focused design activity, adding budgetary constraints, and providing an opportunity for student reflection.
\end{abstract}

Keywords: Engineering design, CEAB graduate attributes, intended learning outcomes, iterative design, first-year students

\section{INTRODUCTION}

The ability to perform engineering design is a critical skill that all engineering students are expected to learn, master, and demonstrate in an undergraduate engineering program. In Canada, design is one of the twelve engineering attributes assessed by the Canadian Engineering Accreditation Board (CEAB) [1], and successful, strong engineering design likely requires engineering students to demonstrate and apply all of the engineering attributes. While there are many engineering design definitions, the reference definition for this paper is: "engineering design is a systematic, intelligent process in which designers generate, evaluate, and specify concepts for devices, systems, or processes whose form and function achieve clients' objectives or users' needs while satisfying a specified set of constraints" [2].

Successful mastery and application of engineering design is often demonstrated in capstone design courses, which are final-year courses focused on students tackling a large, often real-world design problem in teams [2]. Interested readers are directed to a review by Dutson et al. [3], which focuses on the development, format, and other capstone design considerations. Over the last twenty years there has been an increasing introduction of cornerstone design courses or first-year courses focused on students tackling design problems in teams [2]. In the Systems Design Engineering Department at the University of Waterloo, the cornerstone design project has real-world relevance with less interaction with real-world stakeholders and users, compared to capstone projects, due to the time constraints of a 12-week semester.

Given the added difficulty of real-world design problems and the importance of engineering design as a key CEAB graduate attribute, the Systems Design Engineering Department implemented a Design Days Boot Camp (Design Days) that replaces traditional lectures during the first two days of term. Design Days were run for the first time in Fall 2016 and an overview of the original motivation, intended learning outcomes (ILOs), and activities was presented at CEEA17 [4]. Design Days ran successfully in Fall 2016 and Fall 2017 using the presented format [4] with almost complete participation by first year students across the two days of activities. The original Design Days focused on achieving six ILOs, which will be discussed in Section 2.1, through a two-day, team-based design activity focused on building a launcher with design goals of accurate long-distance launching of a ball and precise ball launching at a shorter distance. A secondary activity focused on introducing students to the Student Machine Shop through safety training and individual keychain fabrication. Students worked closely with faculty, shop staff, teaching assistants, and upper year students throughout the two-days. 
Design Days provides an opportunity for students to experience problem-based learning and achieve hands-on engineering design experience before starting their formal, traditional lectures. Design Days shows students the importance that the department places on engineering design and the integral part it will play throughout their five-year undergraduate program. The focus on iterative design also allows for an experiential learning experience with: a concrete experience (via developing an engineered solution), observation and reflection (via testing and analysis), forming abstract concepts (via critical thinking about redesign options), and active experimentation (via testing the new design) [5]. The focus on hands-on sketching, building/prototyping, and testing allows students to experience the kinesthetic benefits of hands-on design, including generating new ideas, visually communicating design ideas, evaluating ideas, and identifying design flaws [5].

In Spring 2018, the Design Days committee decided to investigate two questions: (1) can Design Days be redesigned to improve achievement of specified ILOs and student engagement, and (2) what CEAB graduate attributes are specifically addressed during Design Days activities.

The result of the redesign, and the answer to the first question, was Design Days 2.0. The rest of this paper will present strong aspects of the original Design Days that were maintained, differences between the original Design Days and Design Days 2.0, and the observed differences in student behaviour. Connections between Design Days activities, ILOs, and CEAB graduate attributes will be discussed. Finally, conclusions and ideas for further iterations on Design Days will be included.

\section{DESIGN DAYS 2.0}

\subsection{Retained: Original Design Days Activities}

The original six ILOs were retained in Design Days 2.0. The last ILO was revised and turned into four separate outcomes, giving a grand total of nine ILOs where students were incentivized to:

1. Demonstrate an understanding of their program of study and related courses, resources, faculty and teaching assistants, and traditions within the department or faculty;

2. Show a sense of community within their program, department, and faculty;

3. Demonstrate an understanding how design and technical courses are related as part of the program;

4. Explain the importance of safety during design activities and recognize important safety standards;

5. Use common machining tools in a machine shop setting;
6. Understand a design problem before attempting to create a solution;

7. Work and communicate in a team environment;

8. Follow a design process and apply iterative design towards the creation of a design solution; and

9. Test design solutions and interpret test results as part of iterative design.

The Student Machine Shop safety training and associated keychain activity were retained with no significant changes. For the keychain activity, greater emphasis was placed on safety, both in pre-activity instruction and in operational protocol while the activity was conducted. The launcher design activity was also retained but shortened from a twoday activity to a half-day activity with focus on only one of the two design objectives: precise ball launching at a shorter distance.

The involvement of faculty, shop staff, teaching assistants, and upper year students was retained. The awards provided to high-achieving design teams was also retained and provided students with motivation to succeed and maximize the performance of their design solutions.

\subsection{Improved: New Design Days Activities}

One of the driving forces behind Design Days 2.0 was to provide a greater variety of activities, design experiences, and learning opportunities for students to increase their engagement and achieve a more in-depth, well-rounded introduction to engineering design and their program of study. As such, five different activities were included in Design Days 2.0: three team-based design activities - launcher, cellphone drop, and drawbots along with the individual Student Machine Shop keychain activity, and an individual written communication (Comm.) activity. All three design activities were done in different teams.

The Design Days 2.0 launcher activity was described briefly in 1.0. Students were placed in teams of ten students and tasked with creating a 'launcher' that would launch rubber balls onto a target. The target had various scoring regions based on the precision of the launch. Students were encouraged to test their design before final scoring and had to explain revisions/improvements to their design before testing again. Final score was the total score obtained until the ball missed the target three times. In the event of a tie, the winning team would be decided based on achievement of a stated requirement: minimizing the floor space requirements — width and depth — of the launcher.

For the cellphone drop activity, students were placed in small teams of four to five students and were tasked with creating a device that would allow a cellphone to drop safely to the ground from a height of up to six stories. Students were provided with a simple free-body diagram of the involved forces, the equations for force of gravity and drag force, and drag coefficients for simple shapes. 
Students were instructed to spend the first twenty minutes discussing and sketching potential solutions. During the next phase, students built and tested their solutions. Testing was staged with the testing starting with no cellphone, then progressing to a durable phone, and finally to a smartphone with an accelerometer app. The accelerometer app recorded the maximum acceleration on each axis during the drop with lower accelerations indicating better performance. All of these tests were performed with a one floor drop. The best two teams in each session tested their design with a six-floor drop.

For the drawbots activity, students worked in small teams of four to five students that were different from the cellphone drop teams. Each student built their own drawbot to participate in two team challenges: (1) have the fastest drawbot to completely leave a circular area from a starting point at the center of the circle and (2) create a visually interesting piece of artwork using all of the team's drawbots. The basic design of the drawbot, also known as a scribble-bot [6], consists of a battery and DC motor, an eccentric mass attached to the motor that causes vibration, markers that serve as legs and draw the lines and patterns, and a cup that acts as the body of the device to which other pieces are secured (see Figure 1). Students are encouraged to think about how all of the components come together to form a simple system that can produce complex behaviours.

For the written communication activity, students were asked to communicate what future courses in their program they find most interesting and justify their answer. This activity was inspired by a similar activity in one of the firstyear communication courses, and it was found by the course instructor to be useful in terms of motivating students to think about their long-term learning objectives. Students were also asked to answer an anonymous survey around their apprehension and excitement about becoming an undergraduate engineering student.

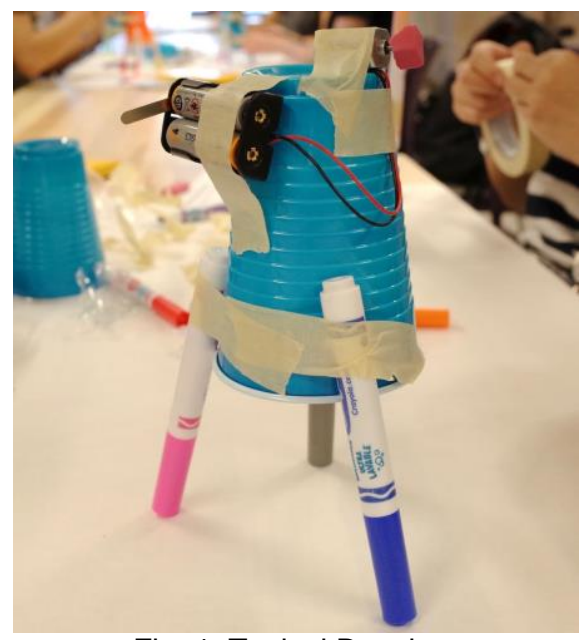

Fig. 1. Typical Drawbot.

\subsection{Scheduling Design Days 2.0}

The management and scheduling of Design Days 2.0 was a design project in and of itself with five activities and 185 first year students from both the Systems Design Engineering (SYDE) and Biomedical Engineering (BME) programs. Students were divided into eight groups consisting of a random mix of SYDE and BME students with approximately 23 students per group. Rotating all of the students through the five activities required careful planning and use of five different spaces on campus across two days. The Schedule for Day One is presented in Table 1 and the schedule for Day Two is presented in Table 2. The use of multiple spaces across the two days helped the students familiarize themselves with some key buildings and spaces where they attended classes and built prototypes during their first term.

Table 1: Design Days 2.0 - Day One Schedule

\begin{tabular}{|c|c|c|}
\hline Time & Main Activity & $\begin{array}{l}\text { Keychain/ } \\
\text { Comm. }\end{array}$ \\
\hline $\begin{array}{l}9: 00- \\
10: 00\end{array}$ & $\begin{array}{l}\text { Welcome and Introduction } \\
\text { by Chair and Undergraduate } \\
\text { Chairs }\end{array}$ & N/A \\
\hline $\begin{array}{l}10: 00- \\
11: 30\end{array}$ & \multirow{2}{*}{$\begin{array}{l}\text { 1\&2: Keychain / Comm. } \\
\text { 3\&4: Drawbots } \\
\text { 5\&6: Launcher } \\
\text { 7\&8: Cellphone Drop }\end{array}$} & $\begin{array}{l}\text { 1: Keychain } \\
\text { 2: Comm. }\end{array}$ \\
\hline $\begin{array}{l}11: 30- \\
1: 00\end{array}$ & & $\begin{array}{l}\text { 1: Comm. } \\
\text { 2: Keychain }\end{array}$ \\
\hline $\begin{array}{l}1: 00- \\
2: 00\end{array}$ & Lunch & N/A \\
\hline $\begin{array}{l}2: 00- \\
3: 30\end{array}$ & \multirow{2}{*}{$\begin{array}{l}\text { 1\&2: Cellphone Drop } \\
\text { 3\&4: Keychain/Comm. } \\
\text { 5\&6: Drawbots } \\
\text { 7\&8: Launcher }\end{array}$} & $\begin{array}{l}\text { 3: Keychain } \\
\text { 4: Comm. }\end{array}$ \\
\hline $\begin{array}{l}3: 30- \\
5: 00\end{array}$ & & $\begin{array}{l}\text { 3: Comm. } \\
\text { 4: Keychain }\end{array}$ \\
\hline
\end{tabular}

Table 2: Design Days 2.0 - Day Two Schedule

\begin{tabular}{|c|c|c|}
\hline Time & Main Activity & $\begin{array}{c}\text { Keychain/ } \\
\text { Comm. }\end{array}$ \\
\hline $\begin{array}{l}9: 00- \\
10: 30\end{array}$ & \multirow{2}{*}{$\begin{array}{l}\text { 1\&2: Launcher } \\
\text { 3\&4: Cellphone Drop } \\
\text { 5\&6: Keychain/Comm. } \\
\text { 7\&8: Drawbots }\end{array}$} & $\begin{array}{l}\text { 5: Keychain } \\
\text { 6: Comm. }\end{array}$ \\
\hline $\begin{array}{l}10: 30- \\
12: 00\end{array}$ & & $\begin{array}{l}\text { 5: Comm. } \\
\text { 6: Keychain }\end{array}$ \\
\hline $\begin{array}{l}12: 00- \\
1: 00\end{array}$ & Lunch & N/A \\
\hline $\begin{array}{l}1: 00- \\
2: 30\end{array}$ & \multirow{2}{*}{$\begin{array}{l}\text { 1\&2: Drawbots } \\
\text { 3\&4: Catapult } \\
\text { 5\&6: Cellphone Drop } \\
\text { 7\&8: Keychain/Comm. }\end{array}$} & $\begin{array}{l}\text { 7: Keychain } \\
\text { 8: Comm. }\end{array}$ \\
\hline $\begin{array}{l}2: 30- \\
4: 00\end{array}$ & & $\begin{array}{l}\text { 7: Comm. } \\
\text { 8: Keychain }\end{array}$ \\
\hline $\begin{array}{l}4: 00- \\
4: 30\end{array}$ & $\begin{array}{l}\text { Event wrap up. } \\
\text { Announcement of design } \\
\text { winners. }\end{array}$ & N/A \\
\hline
\end{tabular}

\subsection{Student Behaviour and Engagement}

Students were engaged throughout the entire two-days of Design Days 2.0 activities. While formal attendance was not taken, it did appear that the majority of the first-year 
cohort attended both days of activities. Students participated fully in all of the activities, with very few or no students observed leaving in the middle of any activity.

Students asked questions that demonstrated that they were actively thinking about the problem and the best design solution. For example, students asked questions about the drag coefficients and differences between semispherical, parachute-like shapes, their associated drag coefficients, and the impact that these would have on drag force. Students also participated fully in the iterative design aspects of the launcher and cellphone drop activities, with several groups achieving substantial improvements in their built designs during the relatively short three-hour period. The launcher activity had the largest group size, increasing the likelihood of students disengaging from the activity. To manage and reduce this occurrence, faculty and teaching assistants interacted with individual students exhibiting disengaged type behaviours (e.g., phone use) and suggested productive activities (e.g., sketching, research, prototyping) that would contribute to team success. This technique was more effective at ensuring complete team engagement earlier in the day than later. Reduced group size could improve engagement and may be considered in future iterations of Design Days.

\section{CONNECTION TO CEAB GRADUATE ATTRIBUTES}

Engineers Canada has identified twelve graduate attributes that accredited engineering programs must teach and instill in their undergraduate students [1]. These graduate attributes are: a knowledge base for engineering (KB), problem analysis (PA), investigation (Inv), design (D), use of engineering tools (Tools), individual and team work (Team), communication skills (CommGA), professionalism (Prof), impact of engineering on society and the environment (Impacts), ethics and equity (Ethics), economics and project management (Econ), and life-long learning (LL). In general, teaching activities within an engineering program should focus on the development of at least one of these attributes and Design Days is no exception. The connection between ILOs, graduate attributes, and Design Days activities is described for the Original Design Days in Section 3.1 and for Design Days 2.0 in Section 3.2.

\subsection{Original Design Days}

The original Design Days event was framed under six ILOs [4], and therefore the connection to graduate attributes will be discussed with regards to these ILOs.

3.1.1. Demonstrating an understanding of their program of study and related courses, resources, faculty and teaching assistants, and traditions within the department or faculty. Design Days starts with introductions to the program by the Department Chair and
Undergraduate Chair and Program Director. During this time, students are introduced to systems level thinking and the potential for this approach to have impact on addressing large, real-world problems. Design Days activities are also run by key faculty who introduce themselves and discuss the first-year courses they will be teaching in the upcoming semester. The associated graduate attribute is Impact. During this introduction, students should realize that their work can have positive (and possibly negative) societal impacts and the importance of adherence to professional engineering ethics.

3.1.2. Showing a sense of community within their program, the department, and the faculty. Students work with their classmates in large teams of approximately 14 students across two days. They also have to interact with and ask questions to faculty and teaching assistants while completing the Launcher activity. Students are encouraged to find useful materials around campus and re-use them to create their Launchers. The associated graduate attribute is Impact. The emphasis on re-use has a connection to sustainable design. Students are also encouraged to think about the safety when testing their Launchers in a safe manner.

3.1.3. Demonstrating an understanding of how design and technical courses are related as part of the program. As students are completing the Launcher activity, they are incentivized to solve the problem iteratively and systematically rather than using trial-anderror approaches. For instance, they are asked to consider (through observation and measurement) the effects of applied forces, energy, materials properties, performance, and so on. Students are then informed that future courses, such as courses on physics and material science, will teach related concepts and techniques that they can apply to systematically solve practical engineering problems, such as the Launcher activity problem. The associated attribute is KB. Students are incentivized to study specifics, rules, principles and theories of mathematics, natural sciences, engineering fundamentals, and specialized engineering knowledge, and reproduce solutions to new problems.

3.1.4. Explaining the importance of safety during design activities and recognize important safety standards. Students attend a mandatory presentation on safety standards and rules when working in the Machine Shop before completing the Keychain activity. Also, safe launching ranges are laid out for the Launcher activity and the importance of testing only in these zones in order to ensure the safety of their classmates and the public is explained. Each person present near the Launcher ranges is required to wear safety goggles. The associated graduate attribute is Prof. An important part of professionalism is protecting the public. Safe testing and machining practices are important to ensure not only personal safety but the safety of others in the same space. 
3.1.5. Using common machining tools in a machine shop setting. In order to successfully complete the Keychain activity, students use a drill press to drill holes, tap holes, countersink, counterbore, and thread. Students also assemble the keychain based on an assembly drawing. The associated graduate attributes is Tools. Knowledge of machine shop equipment and the ability to use this equipment (i.e., tools) is required to successfully build prototypes, which is expected of students during design courses.

3.1.6. Producing a design solution for a specified problem in a team-based setting by understanding the problem before trying to solve it, following a design process and applying iterative design, and ensuring communication among team members. Over the twodays, students are expected to design a Launcher in groups of 14. Before starting their design, students are asked to discuss the problem details and ask questions from the assigned faculty members and graduate students. After that, they are required to go through a mini-design process including identifying the requirements and constraints, developing potential solutions, building a prototype, testing the prototype, and improving on the prototype. The emphasis is on testing and continual improvement during the two days to ensure that students apply iterative design. Furthermore, members of the group leave at various points during the two-days to complete the keychain activity, motivating communication between team members to catch up on missed design decisions. The associated graduate attributes are PA, Design, Team, and CommGA.

\subsection{Design Days 2.0}

Part of the Design Days 2.0 improvements was modifications to the original ILOs. Therefore, the connection to graduate attributes will be discussed with regards to the revised ILOs.

3.2.1. Demonstrating an understanding of their program of study and related courses, resources, faculty and teaching assistants, and traditions within the department or faculty. In addition to the introductions provided on day one, see 3.1.1, faculty running the various events introduced themselves in a smaller, less formal setting at the beginning of their activities. This made it easier for students to ask questions about that faculty member's courses or other concerns. In addition, clear connections are made between Design Days activities and future courses. For example, the connection between all of the design activities and first year/term design courses. Additionally, the connection between second term physics (dynamics) courses and both the cellphone drop and Drawbots activities is discussed. And additional connection between Drawbots and circuit design is made. See 3.2.3. for more detail. The written communication activity engages students in exploring program courses and selecting one of particular interest to find further information about it. This gives students a better understanding of their program of study. The associated graduate attribute is Impact, which was described in 3.1.1.

3.2.2. Showing a sense of community within their program, the department, and the faculty. In addition to working with classmates and interacting with faculty and teaching assistants (as described in 3.1.2), displaying the Drawbot artistic creations in the Department provides another connection to the department community. Upper year students, staff, and faculty in the department can then appreciate the creative artwork, leading to curiosity and interest about the artwork, Drawbots, and Design Days. The Drawbot activity also shows the potential for societal impact of design through the creation and display of technologically created artwork. In the Drawbot activity, students disassemble their drawbots for re-use by the next group of students. Students are also expected to clean up and prepare their stations for the next students as a way of respecting their classmates and general workspaces. Reuse is also encouraged in the Launcher and Cellphone Drop activities, with instructors encouraging students to use materials from previous sessions to create their own devices. In the communication activity, students are also asked to answer an anonymous survey around how they feel about their upcoming new undergraduate experience. The faculty member shares experiences of former students, allowing students to start developing a sense of community. The associated graduate attribute is Impact.

3.2.3. Demonstrating an understanding of how design and technical courses are related as part of the program. One of the focuses of Design Days 2.0 was to strengthen the connection between Design Days activities and course content. In the Launcher activity, faculty discuss the connection between this activity and BME/SYDE161 (first-term design course) through explaining the design process with a particular focus on requirements and testing. The assigned faculty members and graduate students frequently remind students of the activity learning objectives and discuss requirements and constraints with the groups. In the Cellphone Drop activity, faculty discuss the connection between this activity and BME/SYDE161 (first-term design course) through explaining the design process with a particular focus on sketching and testing with acceleration data. A connection is also made to BME/SYDE182 (secondterm physics course) through free-body diagrams, equations, and drag coefficients. For the Drawbots activity, students are introduced to the concept of centrifugal forces, which is a fundamental physics concept, through the vibration of the eccentric mass and subsequent movement of the drawbot (BME/SYDE182). A free-body diagram explaining the involved forces is provided on a handout. In addition, the DC motor and battery represent a basic mechatronic system, with connections to SYDE283 (third- 
term physics course) and BME294 (fourth-term circuits course). Students are introduced, through a handout and instructor explanation, to DC motor schematics, and electro-magnetism principles that explain the workings of a DC motor. In the written communication activity, the connection to BME/SYDE101 (communications course) is made through explaining the importance of effective communication for engineers. The associated graduate attribute is KB. While a high level of technical detail could not be included given the limited time frame, students are introduced to several important engineering concepts, which would prepare them for the in-depth learning that occurs during their first-year of engineering studies.

3.2.4. Explaining the importance of safety during design activities and recognize important safety standards. The machine shop safety training remained the same in Design Days 2.0. In addition to the safe testing space for the Launcher activity, a safe testing space is setup and explained to the students for the Cellphone Drop activity. This is particularly important for the six-floor drop test. Students are instructed to never test a device from any height except in the designated area and only with permission from faculty or teaching assistants who supervise the testing area. The associated graduate attribute is Prof, with an emphasis on ensuring public safety when conducting real-world testing.

3.2.5. Using common machining tools in a machine shop setting. The approach to achieving this ILO and the associated graduate attribute did not change in Design Days 2.0.

3.2.6. Understand a design problem before attempting to create a solution. The approach to understanding the problem before solving it remained the same for the Launcher activity, although it was shortened to accommodate for the reduced timeline. For the Cellphone Drop activity, students had to consider the problem and sketch possible solutions for 20 minutes before entering the build phase of the project. While this may seem like a short period of time, it was just long enough to elicit an 'uncomfortably long' experience for the students who have never experienced the engineering design process before. For the Drawbots activity, students were encouraged to first build up small fundamental components to understand how they functioned, before creating a final design. Figuring out "how it works" is part of the early prototyping and design process required before deciding upon a full solution. The associated graduate attribute for these activities is Design. An important part of engineering design is understanding a problem before trying to solve it and thinking of multiple design solutions and then proceeding with the best option(s). The different strategies employed across the three design activities encourages students to think about design from an engineering perspective.
3.2.7. Work and communicate in a team environment. In Design Days 2.0, students are expected to work in three different groups of different sizes (four to ten students) to successfully achieve design goals in a relatively short period of time. This requires students to effectively work and communicate with each other. Students often had to communicate with faculty and teaching assistants to successfully achieve design goals. In the Drawbots activity, students contribute individual work to ensure the success of the team at achieving artistic and movementbased objectives as both an individual and team challenge are a part of the activity. The associated graduate attribute is Team. The assigned teams are a mix of SYDE and BME students, ensuring that teams are multi-disciplinary. Experiencing three different group dynamics, work strategies, and potential leadership/role delegations across the two-days provides for a richer and more varied team experience for students compared to the one group experience provided in the original Design Days.

3.2.8. Follow a design process and apply iterative design towards the creation of a design solution. In all three design activities, students are encouraged to iterate on their design solution to improve the performance of their prototypes. This iteration is based on testing their prototypes/design solutions, which will be discussed in 3.2.9. In the Launcher activity, students iterate on their design to improve its precision in order to achieve better and more consistent scoring in the testing/scoring area. In the Cellphone Drop activity, students iterate on their design to minimize the cellphone recorded accelerations during a one-floor drop. In the Drawbots activity, students iterate on their design to minimize the time it takes for the Drawbot to leave a set, circular area and maximize artistic outputs. The associated graduate attribute is Design. The iterative design process is a common and successful approach to engineering design that is taught in first-year and upper-year design courses. Students are introduced to this design approach through the hands-on design activities in Design Days 2.0.

3.2.9. Test design solutions and interpret test results as part of iterative design. Incorporating additional testing opportunities into Design Days was an important focus of improvement when developing Design Days 2.0. The original testing within the Launcher activity was retained. Testing is incorporated into the Drawbots activity with stop-watch based assessment of the time taken for the Drawbots to leave a set circular area, giving the students a consistent metric to assess current performance and improvements to their Drawbot design and assembly. In the Cellphone Drop activity, students make progress in their testing from no cellphone, to sturdy cellphone, to test cellphone. This encourages students to think not only about the safety of the public but also of the equipment involved in testing. The test cellphone records accelerations during the one-floor drop, giving a consistent metric upon which 
students could assess the improvement of their design solutions/prototypes. The associated graduate attribute is Inv. Students must appropriately analyze and interpret the testing data to make appropriate changes to their design solutions to improve performance.

\section{INSTRUCTOR REFLECTIONS}

Instructors observed students throughout the two-day boot camp to evaluate student engagement, achievement of learning objectives, and changes in student behaviour between the original Design Days and Design Days 2.0.

In terms of student engagement, it appeared that almost all the students stayed for the entire two days with more than $90 \%$ of students picking up their completion certificate at the end of the second day. Since the event is voluntary and has no impact of grades, this is an important indicator for lifelong learning. The overall engagement was similar to the Fall 2017 Design Days where 90\% of students picked up the certificate. In addition, almost no students were observed disengaging with the activities by going on their phones while the rest of their team worked on the activity. However, on the second day, some teams did finish early and spent time on their phones after their build was complete. This was observed predominantly in the Cellphone Drop, and Launcher activities. Overall, students appeared to be actively engaged with the various activities.

Students appeared to be engaging with the activities in a way that allowed them to meet the intended learning outcomes. For the activities involving team work, students seemed to integrate and participate well with each team. All teams were able to successfully complete the activities by communicating with each other, iterating, and testing their designs. Students demonstrated that they were engaging with the engineering concepts presented by the instructors. For example, in the Cellphone Drop activity, some students asked follow-up questions about the drag coefficients and their related shapes and how those would impact the equations in the free-body diagram.

There were some observed behaviours that indicated areas for improvement. Students appeared to struggle with the sketching activity during the Cellphone Drop activity. Most teams spent the majority of this time discussing ideas and concepts with very little sketching and most teams also felt they had finished this activity before the full twenty minutes had elapsed. This may indicate the need for brief instruction on the advantages and methods for sketching ideas and concepts before beginning this activity. Additionally, the creativity of designs was highest during the first session. This could be due to students becoming tired or less engaged as the two-days continued. It could also be because designs from the first session were put to the side and not disposed of or disassembled before the second session started. The availability of completed designs may have led to design fixation: teams replicating ideas instead of creating their own, unique concepts. The disposal or break-down of design for re-use at the end of each session could help with this issue.

Some changes were observed in student behaviour between the original Design Days and Design Days 2.0. The main change in student behaviour was an increase in iterative design, testing, and asking follow-up questions during Design Days 2.0. This may indicate that the design improvements to Design Days 2.0 have increased student engagement.

\section{CONTINUAL IMPROVEMENT}

As previously mentioned, engineering design is iterative process of incremental improvements. This is also our philosophical approach to Design Days. Design Days 2.0 presents a summation of ideas for incremental improvements that were generated, suggested, and implemented during the two years since Design Days Boot Camp began in Fall 2016. The faculty committee in charge of Design Days Boot Camp has already started the process of gathering new ideas for incremental improvements that will be implemented in future Design Days. Some plans for Design Days 3.0 include changing one of the design activities to focus on a software application, strengthening the connection to course content, incorporating budgeting, and strengthening the learning based on the teamwork experience.

Currently, the Design Days design activities focus primarily on physical design with some circuit design occurring in the Drawbots activity. In order to provide a more well-rounded design experience, a software-focused design activity would be a useful addition that would necessitate removing one of the other design activities. This software-focused design activity could require students to create a virtual environment and ask classmates to experience the developed environment.

While the connection to course content was strengthened in Design Days 2.0, there is potential for further improvements in this area. Students could be asked to actively engage with the technical material presented during the various activities. This could be done by changing provided handouts to a workbook experience. For example, students could be asked to identify the differences between written requirements and constraints for the Launcher activity or solve the drag force equation for a hypothetical solution using different basic shapes for the Cellphone Drop activity. This would strengthen the graduate attribute: KB.

Some materials were provided to the students when building the Launcher and Cellphone Drop activity at no cost. These materials included: craft sticks, garbage bags, pipe cleaners, etc. Effort was made to ensure that students were not limited in the materials available to them. However, unlimited materials is not realistic in the realworld and may reduce the necessity for students to think 
creatively to overcome the limitation of scarce material resources. Therefore, students could be provided with a budget to spend on materials for the Launcher and Cellphone Drop activity with more useful materials (e.g., large garbage bags for the Cellphone Drop activity) costing more than less obviously useful materials (e.g., pipe cleaners). This would require students to consider budgetary constraints in their designs (a typical engineering constraint), think creatively, and achieve success within their means. This would also allow for the incorporation of an additional graduate attribute: Econ.

Finally, while the expansion of the team experience to include three different teams of different sizes improved the learning opportunity focused on the teamwork related ILO (see 3.2.7) and the Team graduate attribute, this learning could be enhanced with a reflection activity. At the end of the second day, students could be asked to reflect on their team experiences in an open discussion or as part of a homework-style written activity. This reflection would focus on asking students to identify characteristics of their teams that improved the working/team dynamic, characteristics that worsened the working/team dynamic, and what they could do in future team experiences to help to promote a positive, productive team dynamic.

In addition to reflection activities, achievement of graduate-attribute-related goals could be assessed through pre- and post-Design Days questionnaires that focus on assessing (at a conceptual level) students' comprehension of graduate-attribute-related concepts. For example, students could be asked to provide characteristics of strong teamwork and sketching or how technical knowledge can be incorporated into the design process.

\section{CONCLUSION}

Design Days Boot Camp was originally conceived in Fall 2016 and successfully ran for two years. This year, substantial improvements were made to Design Days Boot Camp resulting in Design Days 2.0. These changes included adding two additional design activities and a writing activity, strengthening the connection with first year course content, and providing a greater variety of team experiences. The strong connection of Design Days activities to ILOs and graduate attributes shows that meaningful learning can be achieved, at an introductory level, towards understanding engineering design and the creation of competent, skilled professional engineers. The ILO and graduate attribute focused description of Design Days may also assist other departments in creating their own design boot camps.

Design Days 2.0 seemed to increase student engagement and involvement with most if not all of the students staying for the full two days of activities. Design Days Boot Camp in its original and improved form provided a meaningful introduction to engineering design, allowing design instructors to focus on applying engineering design to complex, real-world problems in the first term of engineering studies.

Design Days 2.0 inspired ideas for further improvements including the incorporation of a softwarefocused design activity, further strengthening the connection to course content, the inclusion of budgetary constraints, and student reflection on team activities.

\section{Acknowledgements}

We would like to thank Carolyn MacGregor and Thomas Willett for their substantial contributions and involvement with the creation of the original Design Days Boot Camp. We would also like to thank the Department of Systems Design Engineering for their support of Design Days both through the provision of funding and the willingness of the Department Chair, Paul Fieguth, and Undergraduate Chairs, Carolyn MacGregor and Maud Gorbet, to support this two-day event and speak during the introduction and event wrap up. We would also like to thank Reem Roufail for her advice concerning the connection of graduate attributes to Design Days activities. Finally, we would like to thank colleagues from Engineering Machine Shop, Ideas Clinic, and Faculty of Engineering, for their contribution to Design Days planning, scheduling, and execution.

\section{References}

[1] Engineers Canada, "Canadian Engineering Accreditation Board: 2018 Accreditation Criteria nad Procedures," Nov. 2018.

[2] C. L. Dym, A. M. Agogino, O. Eris, D. D. Frey, and L. J. Leifer, "Engineering Design Thinking, Teaching, and Learning," Journal of Engineering Education, vol. 94, no. 1, pp. 103-120, Jan. 2005.

[3] A. J. Dutson, R. H. Todd, S. P. Magleby, and C. D. Sorensen, "A Review of Literature on Teaching Engineering Design Through Project-Oriented Capstone Courses," Journal of Engineering Education, vol. 86, no. 1, pp. 17-28, Jan. 1997.

[4] I. Ivkovic, T. L. Willett, M. J. Borland, and M. Gorbet, "Design days boot camp: enhancing student motivation to start thinking in engineering design terms in the first year," 1, 2017.

[5] G. Lemons, A. Carberry, C. Swan, L. Jarvin, and C. Rogers, "The benefits of model building in teaching engineering design," Design Studies, vol. 31, no. 3, pp. 288-309, May 2010.

[6] N. B. Sardone, "Building Bots to Develop Systems Thinking," Science Scope, vol. 40, no. 5, p. 33, Jan. 2017. 